\title{
Aplicabilidade do Tidal Model por enfermeiros em serviços de saúde mental: uma revisão de escopo
}

Applicability of the Tidal Model by nurses in mental health services: a scope review

Aplicabilidad del Tidal Model por enfermeras en los servicios de salud mental: una revisión del alcance

Recebido: 27/12/2021 | Revisado: 02/01/2022 | Aceito: 08/01/2022 | Publicado: 11/01/2022

José Leandro Ramos de Lima

ORCID: https://orcid.org/0000-0001-9773-7049 Universidade Federal de Alagoas, Brasil

E-mail: leandroramosdelima@ hotmail.com

Helcimara Martins Gonçalves

ORCID: https://orcid.org/0000-0001-6548-6652

Universidade Estadual de Ciências da Saúde de Alagoas, Brasil

E-mail helcimara@hotmail.com

Flaviane Maria Pereira Belo

ORCID: https://orcid.org/0000-0003-2577-6748

Universidade Federal de Alagoas, Brasil

E-mail: flavi_belo@hotmail.com

Leilane Camila Ferreira de Lima Francisco

ORCID: https://orcid.org/0000-0003-4945-8939

Universidade Federal de Alagoas, Brasil

E-mail: leilanecamila_@hotmail.com

Lucas Kayzan Barbosa da Silva

ORCID: https://orcid.org/0000-0003-0081-1068

Universidade Federal de Alagoas, Brasil

E-mail: lucaskayzan@gmail.com

Nemório Rodrigues Alves

ORCID: https://orcid.org/0000-0002-8821-872X

Universidade Federal de Alagoas, Brasil

E-mail: nemorio_rodrigues@hotmail.com

Maria Zélia de Araújo Lessa Santos

ORCID: https://orcid.org/0000-0003-3879-7695

Universidade Estadual de Ciências da Saúde de Alagoas, Brasil

E-mail: zelialessa@yahoo.com.br

Maria Cicera dos Santos de Albuquerque

ORCID: https://orcid.org/0000-0002-5230-3447

Universidade Federal de Alagoas, Brasil

E-mail: cicera.albuquerque@eenf.ufal.br

\begin{abstract}
Resumo
Objetivo: realizar o mapeamento da literatura acerca da aplicabilidade do Tidal Model por enfermeiros em serviços de saúde mental. Método: Revisão de escopo a qual seguiu as recomendações do Manual de Evidências do Instituto Joanna Briggs, utilizada a estratégia para elaboração da pergunta de pesquisa do tipo Pessoa, Conceito, Contexto (PCC), teve como questão de pesquisa: como se dá a aplicabilidade do Tidal Model por enfermeiros em serviços de saúde mental? Utilizados os termos de busca 'nursing', 'mental health' e 'Tidal Model'. Adotado fluxo de pesquisa proposto pelo PRISMA-ScR. Coleta realizada em agosto de 2021, foram selecionados 12 artigos a partir dos periódicos indexados nas bases de dados PUBMED, CINAHL, Web of Science, SCOPUS e LILACS. Resultados: as informações extraídas dos estudos permitiram a construção das categorias: contexto de aplicação; perfil do usuário do serviço; ações para implantação institucional; desafios para aplicabilidade; cuidados de enfermagem; relacionamento Enfermeiro-Pessoas e resultados do cuidado. Conclusão: O Tidal Model tem sua aplicação de acordo com a natureza da necessidade que a pessoa apresenta, obedecendo as três dimensões que a compreendem. Trata-se de teoria versátil que se adequa ao contexto do serviço. A nível institucional, sua implementação deve obedecer a processos para a incorporação dos fundamentos que norteiam a prática. Ainda são escassos os estudos referentes à aplicabilidade desta teoria no contexto dos serviços de saúde mental brasileiros.
\end{abstract}

Palavras-chave: Enfermagem; Saúde mental; Teoria da maré; Teoria de enfermagem; Cuidado de enfermagem. 


\begin{abstract}
Objective: to map the literature on the applicability of the Tidal Model by nurses in mental health services. Method: Scope review which followed the recommendations of the Joanna Briggs Institute Evidence Manual, using the strategy for preparing the research question of the Person, Concept, Context (PCC) type, had as a research question: how is the applicability of the Tidal Model by nurses in mental health services? The search terms 'nursing', 'mental health' and 'Tidal Model' were used. Adopted research flow proposed by PRISMA-ScR. Collected in August 2021,12 articles were selected from the journals indexed in the PUBMED, CINAHL, Web of Science, SCOPUS and LILACS databases. Results: the information extracted from the studies allowed the construction of the categories: application context; service user profile; actions for institutional implementation; challenges for applicability; nursing care; Nurse-People relationship and care outcomes. Conclusion: The Tidal Model is applied according to the nature of the need that the person presents, obeying the three dimensions that comprise it. It is a versatile theory that fits the context of the service. At the institutional level, its implementation must follow processes for the incorporation of the fundamentals that guide the practice. Studies regarding the applicability of this theory in the context of Brazilian mental health services are still scarce.
\end{abstract}

Keywords: Nursing; Mental health; Tidal model; Nursing theory; Nursing care.

\title{
Resumen
}

Objetivo: mapear la literatura sobre la aplicabilidad del Modelo de Mareas por enfermeras en los servicios de salud mental. Método: Revisión del alcance que siguió las recomendaciones del Manual de Evidencia del Instituto Joanna Briggs, utilizando la estrategia para la elaboración de la pregunta de investigación del tipo Persona, Concepto, Contexto (PCC), tuvo como pregunta de investigación: ¿Cómo es la aplicabilidad del Modelo de Mareas? por enfermeras en los servicios de salud mental? Se utilizaron los términos de búsqueda "enfermería", "salud mental" y "Modelo de mareas". Adoptado el flujo de investigación propuesto por PRISMA-ScR. Recogidos en agosto de 2021, se seleccionaron 12 artículos de las revistas indexadas en las bases de datos PUBMED, CINAHL, Web of Science, SCOPUS y LILACS. Resultados: la información extraída de los estudios permitió la construcción de las categorías: contexto de aplicación; perfil de usuario del servicio; acciones de implementación institucional; desafíos para la aplicabilidad; cuidado de enfermera; Relación enfermera-personas y resultados de la atención. Conclusión: El Modelo de Mareas se aplica según la naturaleza de la necesidad que presenta la persona, obedeciendo a las tres dimensiones que lo componen. Es una teoría versátil que se ajusta al contexto del servicio. A nivel institucional, su implementación debe seguir procesos para la incorporación de los fundamentos que orientan la práctica. Los estudios sobre la aplicabilidad de esta teoría en el contexto de los servicios de salud mental brasileños son aún escasos.

Palabras clave: Enfermería; Salud mental; Teoría de las mareas; Teoría de enfermería; Atención de enfermería.

\section{Introdução}

O cuidado em enfermagem ocorre mediante a execução do Processo de Enfermagem (PE). Este, por sua vez, se desenvolve de maneira sistematizada e tem por objetivo promover mudanças que favoreçam a qualidade de vida e bem-estar, considerando a atual situação do processo saúde-doença da pessoa assistida pelo enfermeiro. Para que se alcance tal resultado, o enfermeiro deve realizar o planejamento terapêutico, o qual deve estar ancorado nas teorias de enfermagem (Teixeira et al., 2018).

Estas, norteiam a prática de cuidado, qualificando-o, uma vez que garantem que todas as ações do enfermeiro serão balizadas por fundamentos filosóficos que abarcam conceitos e pressupostos alinhados com a garantia e promoção da saúde (Teixeira et al., 2018). Entre as teorias de enfermagem, destaca-se o Tidal Model ou “Teoria da Maré”, proposta por Phil Barker, nos anos 2000, enfermeiro e professor de enfermagem psiquiátrica no Reino Unido (Freitas et al., 2020). Trata-se de uma teoria desenvolvida para o cuidado em saúde mental, podendo ser aplicada além da prática da enfermagem, sendo, portanto, considerada um modelo de cuidado interdisciplinar (Monteiro et al., 2015).

Essa teoria utiliza a metáfora da água e do mar de maneira representativa para indicar que a vida é composta por processos contínuos de mudanças. Considera o percurso da vida como uma viagem num oceano de experiências, o qual compreende as alterações do estado de saúde-doença. Assim como o mar, apresenta momentos de estabilidade e calmaria, como de turbulência em meio às "tempestades" que poderão causar instabilidades e até a sensação de que poderá afundar. Nesses momentos, é preciso atracar em um porto seguro para que os reparos necessários sejam realizados, até que a pessoa esteja em condições para retomar a viagem nos oceanos das experiências (Freitas et al., 2020). 
Tais comparações demonstram que o modelo considera a pessoa como protagonista no processo do próprio cuidado, valorizando as experiências vivenciadas por ela. Nessa perspectiva, o enfermeiro é visto como facilitador ou ainda como "salva-vidas" da pessoa que busca ressignificar a sua relação com as experiências geradoras de adoecimento. Nesta teoria, a pessoa é representada em três domínios: o Self ou "Eu" que compreende tudo de mais íntimo que o ser humano apresenta, o Mundo que se refere ao mundo social da pessoa e o Outro que se relaciona com a capacidade de interação com as demais pessoas na vida diária. O Tidal Model considera o cuidado de enfermagem como contínuo, mas que suas ações devem ser direcionadas a um dos domínios. Ao considerar a natureza da necessidade da pessoa, o cuidado poderá ser imediato, quando de intervém em problemas do presente; transicional, que possibilita a transição entre cenários de cuidado; e o desenvolvimental, que é voltado para objetivos a longo prazo (Freitas et al., 2020).

Esse modelo proposto por Barker tem como alicerces a teoria do relacionamento interpessoal de Hildegard Peplau, o modelo de empoderamento no cuidado em saúde mental de Mile Musker e M. Byrne e na teoria do caos (Barker, 2001). Portanto, compreende as situações de stress como oportunidade de crescimento pessoal, principalmente nos mementos de crise em que a pessoa pode representar risco para si e para outros. Nesses casos, o enfermeiro deve estabelecer relação com as experiências vivenciadas pela pessoa para que sejam identificadas as necessidades de cuidado, sendo o relacionamento interpessoal o principal instrumento do profissional (Monteiro, et al., 2015).

Dada a importância e necessidade da prática de enfermagem estar embasada em uma teoria, assim como o Tidal Model se destaca como um modelo para aplicação do PE no contexto dos serviços que atendem às demandas psicossociais, numa lógica oposta ao modelo psiquiátrico, ressalta-se que Freitas et al. (2020) observaram que, no Brasil, a teoria de Barker ainda é pouco conhecida, assim como há pouca produção científica dedicada a sua aplicabilidade nos serviços de saúde mental do país, recomendando o desenvolvimento de pesquisas para aprofundamento de conceitos, aperfeiçoamento e adaptação do modelo aos diversos contextos.

Destaca-se que não foram encontradas revisões sistemáticas relacionadas à aplicação do Tidal Model no cuidado de enfermagem em saúde mental, o que evidencia a necessidade de identificar quais conceitos estão relacionados à utilização deste modelo na assistência de enfermagem no contexto dos serviços da atenção psicossocial. Portanto, este trabalho tem por justificativa preencher as lacunas de conhecimento atuais da literatura, a partir do questionamento: como se dá a aplicabilidade do Tidal Model por enfermeiros em serviços de saúde mental? Portanto, o objeto deste estudo é a aplicabilidade do Tidal Model por enfermeiros em serviços de saúde mental, sendo assim, configura-se como objetivo realizar o mapeamento da literatura acerca da aplicabilidade do Tidal Model por enfermeiros em serviços de saúde mental.

\section{Metodologia}

Trata-se de uma revisão de escopo ou "scoping review", a qual tem por finalidade sintetizar e disseminar resultados de estudos relacionados à determinada temática, sendo seu objetivo mapear o estado da arte de um assunto. Diferentemente das revisões sistemáticas as quais realizam a avaliação das evidências dos diferentes estudos, a revisão de escopo tem como proposta analisá-los de maneira descritiva (Alves, et al., 2021; Ferraz et al., 2020; JBI, 2020; Tricco, et al., 2016).

Esta revisão de escopo cumpriu as recomendações do Manual para Síntese de Evidências do Instituto Joanna Briggs (JBI, 2020). Foi adotado o arcabouço metodológico proposto por Arksey e O’Malley (2005) com as adaptações de Levac, Colquhoun e O'Brien (2010) e Peters et al. (2017), portanto, esta revisão seguiu seis etapas metodológicas: 1 - identificação da questão da pesquisa e objetivo; 2 - identificação de estudos relevantes; 3 - seleção dos estudos conforme critérios definidos; 4 - mapeamento dos dados; 5 - sumarização dos resultados; 6 - apresentação dos resultados, identificando as implicações para a prática profissional, conforme o objetivo da revisão. 
A pergunta ou questão de pesquisa para esta revisão de escopo foi definida a partir da estratégia: População, Conceito, Contexto (PCC). Sendo assim, a população foi composta pelos enfermeiros, conceito sendo aplicação prática do Tidal Model e contexto caracterizado pelos serviços de saúde mental, resultando na seguinte pergunta: Como se dá a aplicabilidade do Tidal Model por enfermeiros em serviços de saúde mental?

A etapa seguinte consistiu em realizar levantamento de possíveis revisões sistemáticas e protocolos que apresentassem relação com a temática e objetivo semelhante a esta revisão. Foram realizadas pesquisas na biblioteca Cochrane e no banco de dados da International Prospective Register os Systematic (PROSPERO), não havendo registros em nenhuma das bases no mês de agosto de 2021. Na sequência, foram definidos Medical Subject headings (MeSH Terms) que possibilitassem o acesso aos artigos referentes à temática, sendo eles 'nursing' e 'mental health'. Apesar do termo 'Tidal Model' não se configurar como MeSH Term, o mesmo foi utilizado para realizar o entrecruzamento. Foram utilizadas as bases de dados de periódicos Literatura Latino-Americana e do Caribe em Ciências da Saúde (LILACS), National Library of Medicine National Institutes of Health (PubMed), Cumulative Index to Nursing and Allied Health Literature (CINAHL), Web os Science e Scopus. Foi utilizada a seguinte estratégia de pesquisa: tidal model AND nursing AND mental health. Foram definidos como critérios de inclusão artigos publicados em periódicos indexados, nos idiomas português, inglês e espanhol e, como critérios de exclusão, artigos que não respondessem à questão de pesquisa. Destaca-se que não foi utilizado o período de publicação como filtro de busca.

Os artigos foram selecionados a partir da execução da estratégia de busca nas bases de dados obedecendo algumas etapas: a leitura do título, ao se identificar aproximação com a temática desta revisão, seguia-se para leitura dos resumos, de maneira semelhante, aqueles que cumprissem com os critérios do estudo, seguiram para a leitura dos artigos na íntegra. Por fim, todos os artigos selecionados, tiveram suas referências revisadas para avaliar a inclusão de alguma delas para análise descritiva. Os artigos foram mapeados no programa Excel ${ }^{\circledR}$ com as seguintes informações: autores, ano de publicação, título, país de origem, periódico, objetivo, metodologia do estudo e trechos com os principais resultados de acordo com o interesse desta revisão. Os artigos duplicados foram excluídos a partir do programa Mendeley. Os artigos foram avaliados seguindo o fluxo e checklist Preferred Reporting Items for Systematic reviews and Meta-Analyses extension for Scoping Reviews (PRISMA-ScR).

Para sumarização dos elementos dos artigos, foi realizada análise qualitativa do conteúdo, sendo criadas categorias que emergiram do aprofundamento da análise dos estudos. Por fim, foi realizada a compilação e comunicação dos resultados com vistas a compreensão geral do conteúdo.

\section{Resultados}

Ao executar a estratégia de busca nas bases de dados, foi obtido um total de 133 resultados. Destes, 30 foram localizados no PUBMED, 41 no CINAHL, 26 no Web of Science, 30 na SCOPUS e 6 na LILACS (Figura 1). Ao realizar cruzamento dos títulos dos periódicos, foram excluídos 77 artigos que se encontravam duplicados em duas ou mais bases de dados. Ao realizar a leitura dos títulos e resumos dos estudos, foram selecionados 23 para a leitura na íntegra, tendo permanecido 12 para compor a amostra final desta revisão de escopo. Após o levantamento das referências contidas nos estudos selecionado, não foram encontrados outros trabalhos que respondessem à pergunta de pesquisa desta revisão. 
Figura 1. Fluxograma para seleção da amostra de estudos, conforme proposto pelo PRISMA-ScR, 2021.

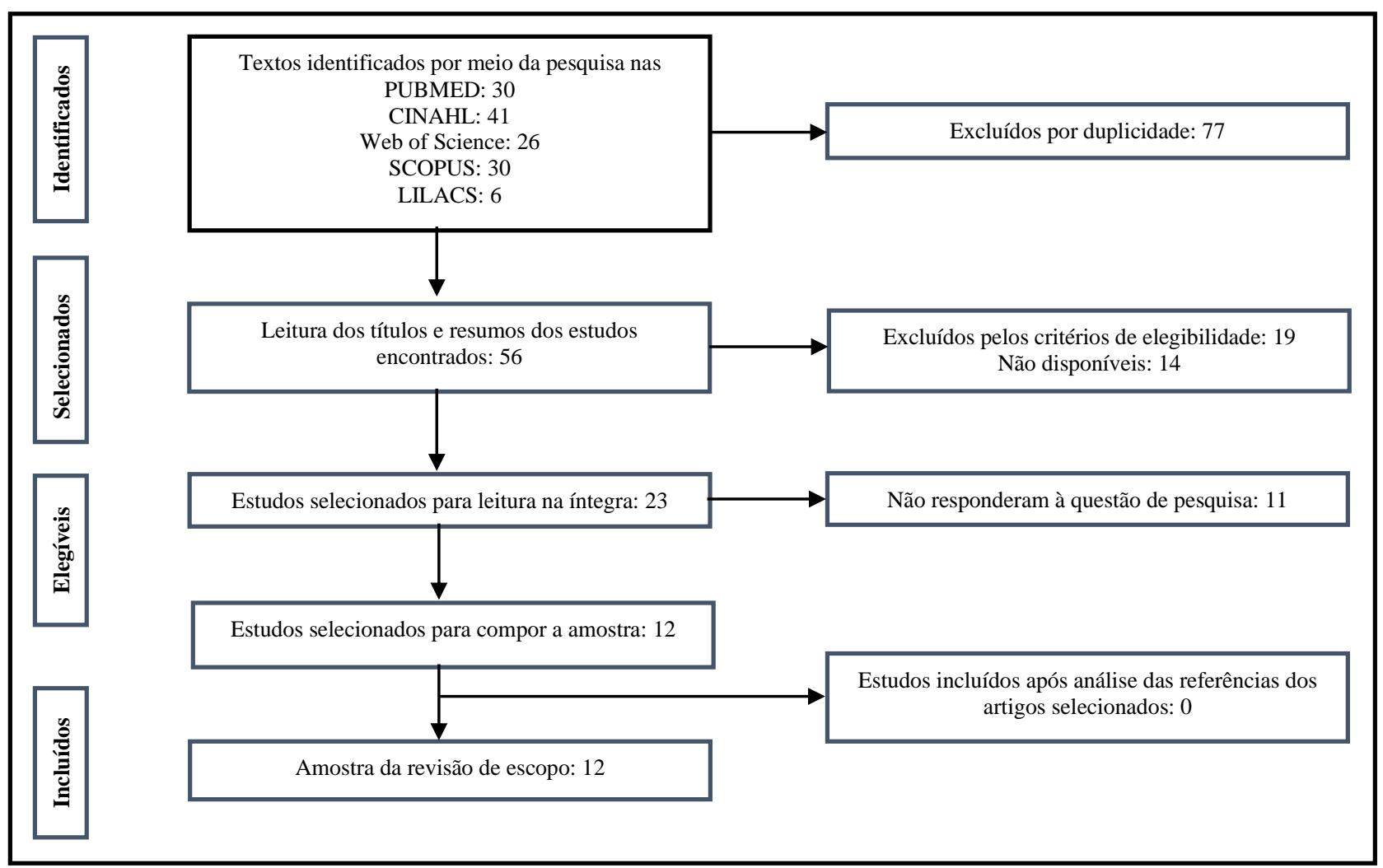

Fonte: Dados da pesquisa (2021).

Após a seleção, foi possível realizar a caracterização e mapeamento dos estudos. Para isso, foi realizado o preenchimento de informações em uma planilha do programa Excel $^{\circledR}$. Para a caracterização, foram utilizadas informações referentes a título, autores, periódico, ano, país, objetivo e metodologia do estudo (Quadro 1). Os artigos foram identificados por classificação a começar de "T1" a "T12". Dos artigos selecionados, 9 foram obtidos via PUBMED, 1 da CINAHL e 1 da Web of Science. Os estudos foram publicados entre os anos 2001 e 2020. Foram obtidos estudos do Brasil, Estados Unidos, Austrália, Turquia, Reino Unido, Canadá, Inglaterra, Irlanda e Escócia, sendo a maior parte dos estudos desenvolvidos por países componentes do Reino Unido $(n=5)$, berço da teoria, seguido pelos Estados Unidos $(n=2)$ e Turquia $(n=2)$. As metodologias aplicadas entre eles variaram entre estudos descritivos, ensaios clínicos, relatos de experiência e relato de caso. Foram identificadas uma revisão integrativa e uma análise crítica, ambas de 2020, alguns dos estudos aqui selecionados foram utilizados por essas revisões. 
Quadro 1. Caracterização dos estudos selecionados referente a título, autores, periódico, ano, país, objetivo e metodologia, 2021.

\begin{tabular}{|c|c|c|c|c|c|}
\hline Título & Autores & Periódico & Ano, país & Objetivo & $\begin{array}{l}\text { Metodologia } \\
\text { do estudo }\end{array}$ \\
\hline $\begin{array}{l}\text { Cuidado de enfermagem em saúde } \\
\text { mental fundamentado o TIDAL } \\
\text { MODEL: revisão integrativa (T1) }\end{array}$ & Freitas et al. & $\begin{array}{l}\text { Revista Brasileira de } \\
\text { Enfermagem }\end{array}$ & $\begin{array}{l}2020 \\
\text { Brasil }\end{array}$ & $\begin{array}{l}\text { Identificar a aplicabilidade do Tida Model, de } \\
\text { Phil Barker, no cuidado de enfermagem em } \\
\text { saúde mental. }\end{array}$ & $\begin{array}{l}\text { Revisão } \\
\text { integrativa da } \\
\text { literatura }\end{array}$ \\
\hline $\begin{array}{l}\text { Analysis of the Tidal Modeland Its } \\
\text { Implications in Late-Life Suicidality } \\
\text { (T2) }\end{array}$ & $\begin{array}{l}\text { Atami e } \\
\text { Lorraine }\end{array}$ & $\begin{array}{c}\text { Nursing } \\
\text { ScienceQuartely }\end{array}$ & $\begin{array}{l}2020 \\
\text { Estados } \\
\text { Unidos }\end{array}$ & $\begin{array}{c}\text { Descrever e analisar criticamente } \\
\text { o Tidal Model no contexto de suicídio em } \\
\text { idosos } \\
\text { e sua utilização prática no atendimento a essa } \\
\text { população. }\end{array}$ & $\begin{array}{l}\text { Revisão e } \\
\text { Análise } \\
\text { crítica }\end{array}$ \\
\hline $\begin{array}{c}\text { The Effect of Tidal Model-Based } \\
\text { Psychiatric Nursing Approach on the } \\
\text { Resilience of Women Survivors of } \\
\text { Viololence (T3) }\end{array}$ & Turgut e Çam & Taylor \& Francis & $\begin{array}{l}2020, \\
\text { Turquia }\end{array}$ & $\begin{array}{c}\text { Examinar o efeito de uma abordagem de } \\
\text { enfermagem psiquiátrica baseada no Tidal } \\
\text { Model sobre a resiliência de mulheres que } \\
\text { sofreram violência. }\end{array}$ & $\begin{array}{l}\text { Ensaio } \\
\text { Clínico }\end{array}$ \\
\hline $\begin{array}{l}\text { Lauching The Tidal Model in na } \\
\text { adult mental health programme (T4) }\end{array}$ & $\begin{array}{l}\text { Fletcher e } \\
\text { Stevenson }\end{array}$ & $\begin{array}{l}\text { Art \& Science } \\
\quad \text { Research }\end{array}$ & $\begin{array}{l}\text { 2001, } \\
\text { Inglaterra }\end{array}$ & $\begin{array}{l}\text { Implementar um novo modelo de enfermagem } \\
\text { psiquiátrica centrada na pessoa } \\
\text { com problemas mentais agudos problemas de } \\
\text { saúde. }\end{array}$ & Estudo Piloto \\
\hline $\begin{array}{c}\text { The Effect of the Psychiatric } \\
\text { Nursing Approach Based on the } \\
\text { Tidal Model on Coping and Self- } \\
\text { steem in people with Alcohol } \\
\text { Dependency: A Randomized Trial } \\
\text { (T5) }\end{array}$ & Savasan e Çam & $\begin{array}{c}\text { Archives of } \\
\text { Psychiatric Nursing }\end{array}$ & $\begin{array}{l}2017, \\
\text { Turquia }\end{array}$ & $\begin{array}{c}\text { Determinar o efeito da abordagem de } \\
\text { enfermagem psiquiátrica com base em } \\
\text { no Tidal Model sobre enfrentamento e auto- } \\
\text { estima em pessoas com dependência de álcool }\end{array}$ & $\begin{array}{c}\text { Ensaio } \\
\text { Clínico } \\
\text { Randomizado }\end{array}$ \\
\hline $\begin{array}{l}\text { Lauching the Tidal Model: } \\
\text { evaluating the evidence (T6) }\end{array}$ & $\begin{array}{l}\text { Gordon, Morton } \\
\text { e Brooks }\end{array}$ & $\begin{array}{l}\text { Journal of } \\
\text { Psychiatric and } \\
\text { Mental Health } \\
\text { Nursing }\end{array}$ & $\begin{array}{l}2005 \\
\text { Reino } \\
\text { Unido }\end{array}$ & $\begin{array}{l}\text { Contribuir com as discussões sobre o tipo de } \\
\text { evidência gerada pela prática para a tomada de } \\
\text { decisão da implementação do Tidal Model }\end{array}$ & $\begin{array}{l}\text { Relato de } \\
\text { caso }\end{array}$ \\
\hline $\begin{array}{l}\text { Sustaining a culture of practice } \\
\text { development in an acute adolescent } \\
\text { inpatient mental health unit (T7) }\end{array}$ & Vella et al. & $\begin{array}{l}\text { Journal of Child and } \\
\text { Adolescent } \\
\text { Psychiatric Nursing }\end{array}$ & $\begin{array}{l}\text { 2017, } \\
\text { Australia }\end{array}$ & $\begin{array}{l}\text { Detalhar os processos, pessoas, recursos e } \\
\text { relacionamentos que permitiram a } \\
\text { implementação bem-sucedida de um programa } \\
\text { de desenvolvimento de práticas uma unidade } \\
\text { de saúde mental para adolescentes. }\end{array}$ & $\begin{array}{l}\text { Relato de } \\
\text { experiência }\end{array}$ \\
\hline $\begin{array}{l}\text { Patient-centred care in acute } \\
\text { psychiatric admission units: } \\
\text { reality or rhetoric? (T8) }\end{array}$ & O’Donovan & $\begin{array}{l}\text { Journal of } \\
\text { Psychiatric and } \\
\text { Mental Health } \\
\text { Nursing }\end{array}$ & $\begin{array}{l}2007 \\
\text { Irlanda }\end{array}$ & $\begin{array}{c}\text { Compreender a prática de enfermagem } \\
\text { psiquiátrica } \\
\text { com pessoas que praticam autolesão usando } \\
\text { uma abordagem descritiva qualitativa. }\end{array}$ & $\begin{array}{c}\text { Estudo } \\
\text { descritivo }\end{array}$ \\
\hline $\begin{array}{l}\text { When Still Waters Become a Soul } \\
\text { Tsunami: Using the Tidal Model to } \\
\text { Recover From Shipwreck (T9) }\end{array}$ & $\begin{array}{l}\text { Kilmer e } \\
\text { Tillerson, }\end{array}$ & $\begin{array}{c}\text { Journal of Christian } \\
\text { Nursing }\end{array}$ & $\begin{array}{l}2013, \\
\text { Estados } \\
\text { Unidos }\end{array}$ & $\begin{array}{l}\text { O uso do Tidal Model em um caso tentativa de } \\
\text { suicídio relacionado ao fracasso de tratamento } \\
\text { para infertilidade. }\end{array}$ & $\begin{array}{l}\text { Relato de } \\
\text { caso }\end{array}$ \\
\hline $\begin{array}{l}\text { How the tidal model was used to } \\
\text { overcome a } \\
\text { risk-averse ward culture (T10) }\end{array}$ & Henderson & Art \& Science & $\begin{array}{l}\text { 2013, } \\
\text { Escócia }\end{array}$ & $\begin{array}{l}\text { Descrever o processo de mudança de } \\
\text { princípios na prática de enfermagem em saúde } \\
\text { mental de uma unidade hospitalar. }\end{array}$ & $\begin{array}{l}\text { Relato de } \\
\text { experiência }\end{array}$ \\
\hline $\begin{array}{l}\text { Incorporation of the tidal model into } \\
\text { the interdisciplinar plan of care - a } \\
\text { program quality improvement } \\
\text { Project (T11) }\end{array}$ & Berger & $\begin{array}{l}\text { Journal of } \\
\text { Psychiatric and } \\
\text { Mental Health } \\
\text { Nursing }\end{array}$ & $\begin{array}{l}\text { 2006, } \\
\text { Canadá }\end{array}$ & $\begin{array}{c}\text { Descrever o desenvolvimento e implantação } \\
\text { de Plano de Cuidado Interdisciplinar de um } \\
\text { hospital psiquiátrico no Canadá }\end{array}$ & $\begin{array}{l}\text { Relato de } \\
\text { experiência }\end{array}$ \\
\hline $\begin{array}{l}\text { The Tidal Model of Mental Health } \\
\text { Recovery na Reclamation: } \\
\text { Application in Acute Care Settings } \\
\text { (T12) }\end{array}$ & $\begin{array}{l}\text { Barker e } \\
\text { Buchanan- } \\
\text { Barker }\end{array}$ & $\begin{array}{c}\text { Issues in Mental } \\
\text { Health Nursing }\end{array}$ & $\begin{array}{l}2010, \\
\text { Reino } \\
\text { Unido }\end{array}$ & $\begin{array}{l}\text { Apontar aplicações e processos de cuidado a } \\
\text { partir do Tidal Model, em unidades de } \\
\text { atendimento de casos agudos. }\end{array}$ & $\begin{array}{c}\text { Revisão } \\
\text { integrativa }\end{array}$ \\
\hline
\end{tabular}

Fonte: Dados da pesquisa (2021).

Mediante análise dos artigos, foi possível mapear as informações referentes à aplicabilidade do Tidal Model nos serviços de saúde mental. Para tanto, as informações foram agrupadas em categorias, sendo elas: contexto da aplicação, perfil do usuário do serviço, ações para implantação institucional, Desafios para aplicabilidade, cuidados de enfermagem, relacionamento Enfermeiro-Pessoa, Resultados do Cuidado (Quadro 2). 
Quadro 2. Mapeamento dos estudos selecionados referentes à aplicabilidade do Tidal Model nos serviços de saúde mental, 2021.

\begin{tabular}{|c|c|c|}
\hline CATEGORIAS & ARTIGOS & PRINCIPAIS RESULTADOS \\
\hline $\begin{array}{l}\text { Contexto da } \\
\text { aplicação }\end{array}$ & $\begin{array}{c}\text { T1, T2, T3, T5, } \\
\text { T7, T9, T10, T11, } \\
\text { T12 }\end{array}$ & $\begin{array}{l}\text { Aplicabilidade da teoria Tidal Model em contextos diferentes como o hospitalar, atenção primária, serviços de saúde } \\
\text { mental, centros de recuperação, unidades de atendimento para uso e dependência de álcool e outras drogas e outras } \\
\text { unidades de saúde (T1, T2 T3, T5, T9, T10, T11); } \\
\text { Atendimento a nível hospitalar para casos agudos, a exemplo ideação suicida (T1, T2, T7, T9, T12). }\end{array}$ \\
\hline $\begin{array}{l}\text { Perfil do usuário } \\
\text { do serviço }\end{array}$ & $\begin{array}{l}\text { T1, T2, T3, T7, } \\
\text { T9, T10, T12 }\end{array}$ & $\begin{array}{l}\text { Pessoas em diferentes ciclos de vida e gênero (T1, T2, T7, T9, T10, T12); } \\
\text { Ideação suicida (T2, T9, T12); } \\
\text { Vítimas de violência (T3); } \\
\text { Usos de substâncias psicoativas (T1, T5) } \\
\text { Pessoas vivenciando adoecimento por transtornos mentais (T1, T9, T10, T12). }\end{array}$ \\
\hline $\begin{array}{l}\text { Ações para } \\
\text { implantação } \\
\text { institucional }\end{array}$ & $\begin{array}{l}\mathrm{T} 1, \mathrm{~T} 4, \mathrm{~T} 7, \mathrm{~T} 10, \\
\mathrm{~T} 11\end{array}$ & $\begin{array}{l}\text { Plano de implementação do modelo Tidal (T7, T10, T11); } \\
\text { Implantação do modelo em conjunto com outras áreas de conhecimento (T1, T11); } \\
\text { Redirecionamento da lógica do cuidado (T1, T10, T11); } \\
\text { Sessões para discussão de casos clínicos (T1, T4, T7). }\end{array}$ \\
\hline $\begin{array}{l}\text { Desafios para } \\
\text { aplicabilidade }\end{array}$ & $\mathrm{T} 1, \mathrm{~T} 2, \mathrm{~T} 8, \mathrm{~T} 10$ & $\begin{array}{l}\text { O paradigma do cuidado centrado na ação médica e medicamentosa (T1, T2, T10); } \\
\text { Dificuldade de implantação do Modelo (T8). }\end{array}$ \\
\hline $\begin{array}{l}\text { Cuidados de } \\
\text { Enfermagem }\end{array}$ & $\begin{array}{l}\text { T1, T2, T3, T4, } \\
\text { T5, T7, T8, T9, } \\
\text { T10, T11, T12 }\end{array}$ & $\begin{array}{l}\text { Ações generalizadas } \\
\text { Acesso às necessidades não atendidas, avaliar gatilhos e planejar o cuidado (T1, T2, T3, T4, T5, T7, T8, T9, T10, } \\
\text { T12); } \\
\text { Abordagem inicial a partir da história de vida da pessoa (T3, T12); } \\
\text { Valorização do relacionamento interpessoal (T1, T7, T9, T11); } \\
\text { Tomada de decisão compartilhada (enfermeiro e pessoa) (T7, T12); } \\
\text { Cuidado individual e adaptado (T1, T3, T5, T9, T10, T12); } \\
\text { Avaliação e reavaliação do plano de enfermagem (T4); } \\
\text { Promoção do crescimento pessoal a partir da experiência da crise vivenciada (T2, T12); } \\
\text { Domínio do Eu } \\
\text { Desenvolvimento do plano de segurança individual (T5, T10, T12) } \\
\text { Construção de pontes para gerenciamento do risco (T1, T3, T9, T12); } \\
\text { Dimensões do cuidado: imediato - realizar conversa abeta para acessar sentimentos profundos (T2, T3, T12); } \\
\text { Domínio do Mundo } \\
\text { Atendimento individual (onte-to-one) (T5, T12); } \\
\text { Cópia dos planos de cuidado para o usuário (T4, t12); } \\
\text { Desenvolvimental - explorar o histórico de vida (a exemplo, tentativas de suicídio) (T2, T3, T12); } \\
\text { Domínio do Outro } \\
\text { Atividades grupais (T3, T12); } \\
\text { Transicional - valorizar suporte comunitário, familiar, amigos e preparar para o retorno à comunidade (T2, T3, T12); } \\
\text { Orientação e estímulo para autonomia e tomada de decisão pela própria pessoa (T3, T12); } \\
\text { Estímulo para o fortalecimento dos recursos internos (espirituais) e externos (de relacionamento interpessoal) (T3); } \\
\text { Processos de reabilitação a partir de modelo prático para exploração das dimensões (T1, T2); }\end{array}$ \\
\hline $\begin{array}{l}\text { Relacionamento } \\
\text { Enfermeiro-Pessoa }\end{array}$ & $\begin{array}{c}\text { T1, T2, T3, T5, } \\
\text { T7, T8, T9, T10, } \\
\text { T11, T12 }\end{array}$ & $\begin{array}{l}\text { O cuidado em saúde mental centrado na pessoa (T1, T2, T9, T10, T11, T12); } \\
\text { Enfermeiro e pessoa buscam explorar juntos a experiência do transtorno mental (T1, T2, T9, T10, T11, T12); } \\
\text { Respeito à cultura e etnia das pessoas em sofrimento (T1, T3, T10); } \\
\text { Empoderamento do enfermeiro e da pessoa (T1, T10, T12); } \\
\text { Valorização da fala da pessoa em sofrimento (T2, T3, T8, T9, T10, T12); } \\
\text { Valorização das habilidades da pessoa e à busca de soluções (T3, T5, T10, T11, T12); } \\
\text { Relacionamento pautado nos } 10 \text { compromissos e nas } 20 \text { competências do Tidal Model (T1, T7, T9 T10); } \\
\text { Encontros que promovam a segurança da pessoa (T3, T12). }\end{array}$ \\
\hline $\begin{array}{l}\text { Resultados do } \\
\text { cuidado }\end{array}$ & $\begin{array}{l}\text { T1, T2, T3, T4, } \\
\text { T5, T6, T9, T10 }\end{array}$ & $\begin{array}{l}\text { Redução de agressão física, violência, assédio (T1, T4, T6, T12); } \\
\text { Redução do uso de substâncias psicoativas (T5); } \\
\text { O usuário do serviço de saúde apresenta perspectiva positiva acerca da assistência prestada (T1. T10); } \\
\text { Satisfação do enfermeiro (T1, T4, T10, T12); } \\
\text { Melhoria nas estratégias de coping e autoestima da pessoa (T1, T3, T5, T9); } \\
\text { Promove melhor avaliação do enfermeiro acerca do quadro clínico, diante da pessoa com ideação suicida (T2, T9); } \\
\text { Aumento da resiliência de mulheres vítimas de violência (T3); } \\
\text { Redução do tempo de internamento e do uso de contenção (T4); } \\
\text { Valorização da prática de enfermagem em saúde mental (T4, T10, T12); } \\
\text { Aproximação do enfermeiro à pessoa (T10). }\end{array}$ \\
\hline
\end{tabular}

Fonte: Dados da pesquisa (2021). 
Na categoria Contexto de aplicação, foram mapeadas as informações acerca das situações em que o Tidal Model foi implementado. Os estudos trazem a aplicação em contextos variados como na atenção hospitalar, atenção primária e outros serviços de saúde mental, entretanto, há destaque para o desenvolvimento do cuidado pautado no Tidal Model nos serviços hospitalares (T1, T2, T9, T10, T11, T12), bem como em situações de atendimento de casos agudo, como em situação de tentativa/ideação suicida (T1, T2, T7, T9).

De maneira semelhante foram selecionadas as informações sobre o perfil do usuário dos serviços os quais recebeu cuidados na perspectiva do Tidal Model. Foram obtidas informações da sua aplicabilidade tanto na população mais jovem (adolescentes) como adultos e idosos, assim como não há distinção de seu uso de acordo com o gênero. Quanto ao motivo da necessidade do cuidado, o uso do Modelo foi direcionado para pessoas vivenciando ideação suicida/tentativa de suicídio, vítimas de violência, uso e abuso de substâncias psicoativas e outros acometimentos por transtornos mentais, como depressão.

No que se refere às informações quanto as ações para implantação institucional, foi observado o desenvolvimento de plano estruturado para implementação do Tidal Model (T7, T10, T11), os quais, em alguns casos, contou com a implantação do modelo em conjunto com outras áreas de conhecimento, além da enfermagem (interdisciplinar), ações de educação continuada para fortalecimento da cultura do Tidal Model para os profissionais por meio de sessões para discussão de casos clínicos (T1, T4, T7). Os estudos trazem a informação de que o uso do Modelo se deu pela busca do redirecionamento da lógica do cuidado (T1, T10, T11).

Outros estudos apresentaram os desafios e dificuldades para aplicabilidade do Tidal Model, sendo o principal deles a mudança do paradigma médico-centrado que, por sua vez, possui uma perspectiva medicalocênctrica e difundida na sociedade (T1, T2, T10). Um dos estudos (T8) apresentou dificuldade para incorporar a Teoria à prática dos profissionais, havendo resistência de seu uso por parte dos profissionais.

Entre os cuidados de enfermagem mapeados nos estudos para a prática profissional embasada no Tidal Model, os mais mencionados foram o acesso às necessidades não atendidas da pessoa, avaliar gatilhos e planejar o cuidado (T1, T2, T3, T4, T5, T7, T8, T9, T10, T12), a valorização do relacionamento interpessoal (T1, T7, T9, T11, T12), o cuidado individual adaptado (T1, T3, T5, T9, T10, T12) e a construção de ponte para gerenciamento do risco (T1, T3, T9, T12).

Ressalta-se que outras ações de cuidado baseadas nos princípios de Barker também foram mapeadas como a abordagem inicial a partir da história de vida da pessoa (T3, T12), a tomada de decisão compartilhada (T7, T12), avaliação e reavaliação do plano de enfermagem (T4), realização de atividades grupais (T3, T12), assim como de atendimento individual (sessões one-to-one) (T5, T12), realização d plano de segurança (T5, T10, T12), cópia dos planos de cuidado para o usuário (T4, T12), promoção do crescimento pessoal a partir da experiência da crise ou transtorno vivenciado (T2, T12), o cuidado aplicado à dimensão necessária do momento (imediato, desenvolvimental e transicional) (T2, T3, T12), estímulo para autonomia, tomada de decisões e fortalecimento dos recursos internos e externos (T3) e a busca da reabilitação explorando o cuidado de cada uma das dimensões (T1, T2, T12).

Tendo em vista que Barker propõe uma teoria que tem por base o relacionamento interpessoal, uma das categoria para mapeamento da prática do Tidal Model se dedicou ao relacionamento Enfermeiro-Pessoa, o qual os estudos trazem que esse vínculo acontece por meio do cuidado em saúde mental centrado na pessoa (T1, T2, T9, T10, T11, T12), enfermeiro e pessoa buscam de forma conjunta explorar a experiência do transtorno mental (T1, T2, T9, T10, T11, T12), o profissional respeita a cultura e etnia da pessoa em sofrimento(T2, T3, T8, T9, T10), bem como valoriza as habilidades e a busca de soluções (T3, T5, T10, T11, T12), os encontros entre profissional e pessoa promovem segurança para a pessoa (T3, T12) e são pautados nos 10 compromissos do Modelo e nas 20 competências (T1, T7, T9 T10).

Por fim, a categoria referente aos resultados do cuidado traz as informações dos resultados gerados pela aplicação/incorporação do Tidal Model, sendo elas representativas para profissionais e usuários dos serviços relatados nos 
estudos. Foram observados a redução da agressão física, violência dirigida a outros ou a si mesmo e assédio (T1, T4, T6, T12), redução do uso de substâncias psicoativas (T5), aumento da resiliência de mulheres vítimas de violência (T3), melhoria nas estratégias de coping e autoestima da pessoa (T1, T3, T5, T9), satisfação do enfermeiro (T1, T4, T10, T12), avaliação positiva do usuário do serviço acerca da assistência prestada (T1, T10), amplitude da avaliação do enfermeiro relacionado ao quadro de saúde da pessoa com ideação suicida (T2, T9), redução do tempo de internamento e do uso de contenção (T4), aproximação do enfermeiro à pessoa que precisa de cuidados (T10) e a valorização da prática de enfermagem em saúde mental (T4, T10, T12).

\section{Discussão}

Entre os achados iniciais, observa-se a limitação das publicações relacionadas à teoria. Fato que pode ser associado ao ano em que se deu a sua primeira publicação (Barker, 2001), uma vez que o Tidal Model teve seu desenvolvimento iniciado no ano de 1996, pensado a partir da carência percebida na prática da enfermagem psiquiátrica. O Tidal Model surge a partir da necessidade do estímulo para construção de vínculo e empoderamento no cuidado de saúde mental entre enfermeiro e pessoa cuidada. Trata-se, portanto, de modelo de cuidado em saúde mental focado na promoção do cuidado, prevenção do processo de adoecimento e ressignificação das experiências de vida, o qual foi desenvolvido por enfermeiros especialistas em saúde mental, para a prática de enfermagem nesse contexto. É considerado tanto um modelo quanto uma teoria. Mas, antes de tudo, configura-se também como uma filosofia pragmática a qual impulsiona a prática na direção do processo de recuperação, uma vez que o profissional é estimulado a pensar nas ações necessárias para que o cuidado aconteça neste sentido (Barker \& Buchanan-Barker, 2010).

A teoria foi estruturada de forma conjunta entre profissionais de saúde mental e grupos de usuários sob seus cuidados, foi proposto para que seu uso também ocorra em situações extremamente desafiadoras, como, por exemplo, diante de um episódio psicótico agudo. Foi planejada para que seja executada para além da atenção hospitalar, ao considerar o cuidado na comunidade para pessoas em todas as fases de vida (Barker et al., 2010).

Tais informações são reforçadas pelos dados mapeados nas categorias referentes a 'Contexto de aplicação' e 'Perfil do usuário do serviço', os quais demonstram a versatilidade da aplicação do Tidal Model de acordo com as necessidades que a pessoa apresenta para o profissional. Há exemplo, como os estudos de Jacob et al. (2008) e Cook et al. (2005) que relatam a aplicação do modelo no contexto de unidades de psiquiatria forense.

A aplicabilidade da teoria em contextos diferentes se dá por sua concepção das três dimensões que compreendem a pessoa, o "Eu", o "Mundo" e o "Outro" e o fato de cada uma delas inspirar cuidados específicos. De forma prática, pode-se descrever a dimensão do "Eu" relacionada aos cuidados emergenciais, a qual se propõe a promover segurança para a pessoa, principalmente quando ela pode se tornar risco para si ou para outras pessoas. Momento de interação e valorização da voz, fortalecimento de vínculo e percepções entre enfermeiro salva vidas e pessoa cuidada em naufrágio no oceano de experiências. Enquanto no domínio do "Mundo" a pessoa é estimulada a realizar avaliação holística a partir de sua própria história, aqui se busca a compreensão das experiências vivenciadas e a ressignificação delas. O domínio do "Outro", por sua vez, é proposto quando a pessoa se encontra relutante a se entregar para o processo terapêutico por meio do acompanhamento individual, assim, este domínio propõe o desenvolvimento das habilidades de relacionamento da pessoa a partir de encontros em grupo (Barker et al. 2010).

Foi percebido, no decorrer do levantamento das publicações, que havia quantitativo significativo de estudos que se propuseram a avaliar o arcabouço teórico do Tidal Model. Ao passo que ainda são restritos aqueles que se dedicam a observar as evidências de sua aplicação nos diversos contextos em saúde mental. As informações mapeadas na categoria 'Ações para implantação institucional' enfatizam a necessidade de plano de implantação estruturado e que tenha participação de outras áreas de conhecimento para promoção de saúde mental. Para tanto, assim como executado por Pereira e Coelho (2019), toda 
proposta de mudança da prática de cuidado a nível institucional deve passar por algumas etapas, como reuniões de planejamento, sensibilização dos profissionais, estabelecer uma equipe de trabalho, elaboração de projeto piloto, divulgação e sensibilização da equipe, capacitação dos profissionais, implementação do projeto piloto, avaliação do processo, implantação do projeto para os demais setores e sistematização dos dados e divulgação dos resultados para estabelecimento de fluxos institucionais.

O processo de implantação de uma nova proposta de prática em saúde deve ter sua continuidade garantida por meio de ações de educação permanente, a qual é compreendida como foram de potencializar e qualificar o processo de trabalho em saúde (Pinheiro et al. 2019). No caso da implantação do Tidal Model, a educação permanente se configura como meio de garantir que todos os profissionais envolvidos executem suas ações conforme o proposto pela teoria, e que possam revisitar a prática profissional constantemente.

Tais processos, quando incorporam duas ou mais áreas de conhecimento, possibilitam a interdisciplinaridade e, consequentemente, potencializam o cuidado em saúde. As ações interdisciplinares acontecem por meio do relacionamento e articulação das diferentes formas de saber e prática que trabalham associadas em nome de um bem comum, entretanto, sem deixar de valorizar o conhecimento e relevância de cada profissão (Rocha \& Lucena, 2018). Da mesma forma o Tidal Model reconhece a importância do cuidado interdisciplinar em saúde mental, não cabendo a uma única categoria tomar a responsabilidade de promover saúde, tendo em vista a que cada profissão poderá atender as demandas específicas da pessoa em sofrimento, de formas diferentes. Apesar de ser uma teoria pensada para a prática de enfermagem, pode ser implementada por qualquer profissional de saúde mental (Dias et al. 2020; Barker, 2001).

O Tidal Model propõe, de forma semelhante à reforma psiquiátrica brasileira, modificar o paradigma de cuidado da pessoa com transtorno mental. Para tanto, o profissional deve se apropriar da teoria para que sua prática esteja fundamentada e possua significado tanto para o usuário quanto para o próprio profissional. Assim como a essência da atenção psicossocial, trata-se de uma teoria que implica mudanças teórico-conceituais (Fidelis, 2018). Quando há problemas para incorporação de sentido à prática do profissional que executa o Tidal Model, é possível perceber as dificuldades mapeadas na categoria 'Desafios para aplicabilidade'. A prática fragilizada por necessidade de maior embasamento esbarra no paradigma bem consolidado da cultura psiquiátrica. Além disso, é preciso considerar as limitações citadas pelo estudo T8, o qual menciona a fragilidade metodológica, e resultados que precisam ser interpretados e considerados com cautela.

No que se refere à categoria 'Cuidados de enfermagem' é possível visualizar rol de ações mapeadas, as quais podem ser divididas de acordo com a necessidade específica de uma dimensão, ou aquelas que servem como base e ponto de partida para qualquer intervenção junto à pessoa. Entretanto, todas as ações devem ter como base a construção e fortalecimento da pessoa, permitindo que ela se coloque como foco de todas as ações, com vistas a prestação de cuidado em saúde mental efetivo e de qualidade, como é possível verificar nos itens mapeados nas categorias 'Relacionamento Enfermeiro-Pessoa' e 'Resultados do cuidado'.

De acordo com Barker (2001), a enfermagem tem forte aproximação com teorias baseadas no relacionamento interpessoal, apesar da forte influência positivista que tem sofrido nos últimos anos em busca de intervenções baseadas em evidência. O Tidal Model propõe que o enfermeiro abandone a prática pautada especialmente na técnica para que se aproxime emocionalmente da pessoa que inspira cuidados. Dessa forma, é possível viver a experiência do adoecimento de maneira compartilhada com a pessoa. Ao se tratar dos transtornos mentais, as experiências de sofrimento vivenciado são particulares e únicas, uma vez que envolve diversos significados atribuídos pela pessoa, em relação de ser humano e de estar vivo.

Portanto, para promover o cuidado, Buchanan-Barker e Barker (2008) afirmam que a pessoa é a "chave" que dará norte para o processo de recuperação, propõe que nesta relação Enfermeiro-Pessoa, esta seja a protagonista. Elencam ainda seis pensamentos filosóficos chave a serem respeitados a cada encontro: acreditar na virtude da curiosidade, ao buscar aprender 
sobre os "mistérios" da pessoa; reconhecer o poder da desenvoltura, ao focar nos potenciais recursos da pessoa a serem desenvolvidos; respeitar os desejos da pessoa, e o enfermeiro abandonar o papel paternalista; aceitar o paradoxo da crise como oportunidade para mudança do curso de vida; reconhecer que todas as metas pertencem à pessoa e; a virtude da "elegância" ao buscar meios simples e possíveis de serem aplicados pela pessoa, uma vez que o tratamento psiquiátrico costuma ser complexo e desconfortante.

A Teoria propõe que o profissional assuma papel de auxiliar, possibilitando que a pessoa consiga promover mudanças na própria vida e que, portanto, a assistência não seja pautada exclusivamente no controle e gerenciamento de sintomas (Buchanan-Barker \& Barker, 2008). Tais conceitos, evidenciam a proximidade da teoria com a atual política de saúde mental proposta no Brasil. Entretanto, durante o levantamento dos estudos, foi percebida a ausência de publicações nos periódicos indexados relacionados à execução do Tidal Model no contexto dos serviços de saúde mental brasileiros.

A Reforma Psiquiátrica suscitou transformações no campo da assistência e que demanda que o enfermeiro assuma a postura de agente terapêutico, para tanto, exige sensibilidade e compreensão de conteúdos específicos para apresentar resposta às demandas dos usuários dos serviços de saúde mental (Garcia et al., 2017). Portanto, o Tidal Model se configura como oportunidade para esses profissionais, de se apropriarem de teoria desenvolvida especificamente para esta prática como meio de conferir maior qualidade do cuidado prestado às pessoas em sofrimento mental e, consequentemente, o fortalecimento da Política de Atenção Psicossocial do país.

\section{Conclusão}

Diante do exposto, é possível perceber, por meio do mapeamento da literatura levantada, que a aplicação prática do Tidal Model ocorre de acordo com a natureza da necessidade que a pessoa apresenta ao acessar o serviço de saúde mental. Sendo assim, cabe ao profissional avaliar qual dimensão necessita de suporte para que possa vislumbrar o tipo de cuidado necessário. A nível institucional, é preciso que conjunto de ações sejam desenvolvidas para que a equipe possa incorporar não apenas a prática, mas os fundamentos da teoria. A literatura levantada aponta tanto a versatilidade quanto o impacto positivo da teoria na prática de enfermeiros em diferentes contextos.

Faz-se fundamental destacar que a prática deve estar alinhada com a filosofia proposta, com vistas o cuidado centrado na pessoa em busca do empoderamento da relação Enfermeiro-Pessoa, bem como possibilitar ressignificação a partir da experiência do transtorno vivenciado.

Por fim, ressalta-se que a teoria possui afinidade em seu escopo com a lógica de cuidado em saúde mental no Brasil, entretanto, ainda são poucos os estudos que se debruçam sobre a teoria, no país. Recomenda-se o incentivo de pesquisas na busca da implementação e adaptação desta teoria no contexto dos serviços de saúde mental brasileiros, bem como oportunizar o acesso à teoria a partir do processo de formação acadêmica em enfermagem.

\section{Referências}

Alves, N. R., et al. (2021). Atuação dos profissionais do consultório na rua no âmbito da Atenção Primária à Saúde do Brasil: Uma revisão de escopo. Research, Society and Development, 10(4), 1-13.

Arksey, H. \& O’Malley, L. (2005). Scoping studies: towards a methodological framework. Int. j. soc. res. Methodol, 8, 19-32.

Barker, P. (2001). The Tidal Model: developing an empowering, person-centred approach to recovery within psychiatric and mental health nursing. Journal of Psychiatric and Mental Health Nursing, 8(1), 233-240.

Barker, P. \& Buchanan-Barker, P. (2010). The Tidal Model of Mental Health Recovery and Reclamation: Application in Acute Care Settings. Issues in Mental Health Nursing, 31, 171-180.

Buchanan-Barker, P. \& Barker, P. (2008). The Tidal Commitments: extending the value base of mental health recovery. Journal of Psychiatric and Mental Health Nursing, 15, 93-100. 
Cook, N. R., et al. (2005). The tidal model as experienced by patients and nurses in a regional forensic unit. Journal of Psychiatric and Mental Health Nursing, $12,536-540$.

Dias, M. K. \& Ferigato, S. H. \& Fernandes, A. D. S. A. (2020). Atenção à Crise em saúde mental: centralização e descentralização das práticas. Ciênc. saúde coletiva, 25(2), 595-602.

Ferraz, L. \& Peireira, R. P. \& Pereira, A. M. R. C. Tradução do conhecimento e os desafios contemporânea área da saúde: uma revisão de escopo. Saúde debate, 43 (spe2), 200-216.

Fidelis, A. C. (2018). Sentido do cuidado em saúde mental: sobre a rede de atenção psicossocial do Sistema Único de Saúde (SUS). Trab. educ. saúde, 16(2), $561-582$.

Freitas, R. J. M., et al. (2020). Cuidado de enfermagem em saúde mental fundamentado no TIDAL MODEL: revisão integrativa. Rev. Bras. Enferm., 73(2), 111 .

Garcia, A. P. R. F., et al. (2017). Processo de enfermagem na saúde mental: revisão integrativa da literatura. Rev. Bras. Enferm., 70(1), $220-230$.

Jacob, J. D. \& Holmes, D. \& Buus, N. (2008). Blackwell Publishing Ltd Humanism in forensic psychiatry: the use of the tidal nursing model. Nursing Inquiry, 15(3), 224-230.

JBI, Joanna Briggs Institute (2020). The Joanna Briggs institute reviewers' manual 2020: methodology for JBI scoping reviews. South Australia: The Joanna Briggs Institute.

Levac, D. \& Colquhoun, H. \& O’Brien, K. K. (2010). Scoping studies: advancing the methodology. Implement. Sci, 5(1), 5-69.

Monteiro, A. R. M, et al. (2015). Sistematização da assistência de enfermagem à criança e ao adolescente em adoecimento psíquico. J. res.: fundam. care, $7(4), 1732-1743$.

Pereira, R. A. \& Coelho, C. F. C. (2019). Implantação do acolhimento com classificação de risco na rede hospitalar e seu impacto na atenção primária a saúde. Revista Extensão, 3(1), 179-183.

Peters, M. D. J., et al. (2017). Scoping Reviews. In: Aromataris E, Munn Z, editores. Joanna Briggs Institute Reviewer's Manual. Australia: The Joanna Briggs Institute.

Pinheiro, M. C. M. \& Hypólito, A. L. M. \& Kantorski, L. P. (2019). Educação permanente no processo de trabalho em saúde mental. Journal of Nursing and Health, 9(2), 1-15.

Rocha, E. N. \& Lucena, A. F. (2018). Projeto Terapêutico Singular e Processo de Enfermagem em uma perspectiva de cuidado interdisciplinar. Rev. Gaúcha Enferm, 39, 1-11.

Teixeira, L. A., et. al. (2018). Teoria da Maré: análise pautada na perspectiva de Meleis. Rev. Bras. Enferm., 71(2), 488-494.

Tricco, A. C., et al. (2016) Knowledge synthesis methods for generating or refining theory: a scoping review reveals that little guidance is available. J. clin. Epidemiol, 73, 36-42. 